\title{
First Excited Band of a Spinor Bose-Einstein Condensate
}

\author{
C.G. Bao and Z.B. Li \\ The State Key Laboratory of Optoelectronic Materials and Technologies \\ Department of Physics, Zhongshan University, Guangzhou, 510275, P.R. China
}

\begin{abstract}
The analytical expression of the fractional parentage coefficients for the total spin-states of a spinor N-boson system has been derived. Thereby an S-conserved theory for the spinor BoseEinstein condensation has been proposed. A set of equations has been established to describe the first excited band of the condensates. Numerical solution for ${ }^{23} \mathrm{Na}$ has been given as an example.
\end{abstract}

PACS numbers: 03.75. Fi, 03.65. Fd

In recent years, accompanying the experimental realization of the spinor Bose-Einstein condensation (BEC) 1, 2, 3, 4, 5], corresponding theories based on the mean field theory have been developed 6, 7, 8, 9, 10, 11, 12, 13. The total spin S together with its Z-component are actually conserved in the spinor condensates. Furthermore, the interactions are in general spin-dependent. Therefore, the strength of the mean field $g$ that acts on the macroscopically occupied quantum states might depend on S. In previous theories, the S-dependence of the strength $g$ has not yet been perfectly clarified. A primary attempt along this line was proposed in Ref. 14] where the total spin-states have been introduced and the S-dependence of $g$ has been derived analytically.

The present paper is a continuation of the Ref. 14]. It is noted that the thermodynamical property of condensates at low temperature depends on their low-lying spectra, this is an interesting topic scarcely touched. The first aim of the paper is to generalize the study of Ref. 14 from the ground band (GB) to excited bands. Rigorous total spin-states with good quantum numbers, $S$ and $S_{Z}$, and with a specific permutation symmetry (see below) will be introduced, a set of equations is derived to describe, as a first step, the first excited band (FEB) of the spinor BEC.

When the total spin states are adopted, how to achieve the matrix elements among them is crucial. The second aim of this paper is to develop a general tool, namely the fractional parentage coefficients, to facilitate the theoretical treatment of spinor N-boson systems. The case of ${ }^{23} \mathrm{Na}$ as an example will be studied numerically. Before going ahead, we need some knowledge from few-boson systems.

Let the interaction between a pair of spin-one bosons be $U_{i j}=\delta\left(\mathbf{r}_{i}-\mathbf{r}_{j}\right) O_{i j}, O_{i j}=g_{m} P_{m}+g_{q} P_{q}=c_{0}+$ $c_{2} \mathbf{F}_{i} \cdot \mathbf{F}_{j}$. Where $P_{m}$ and $P_{q}$ are projectors to the two-body spin-states with spin $S_{i j}=0$ and 2 (spin 1 is irrelevant since in this case both the spin wavefunction and the spatial wavefunction must be antisymmetric that prevents a point interaction), respectively, $g_{m}$ and $g_{q}$ are the associated strengths, $\mathbf{F}_{i}$ is the spin operator of particle $i, c_{0}=\left(g_{m}+2 g_{q}\right) / 3$, and $c_{2}=\left(g_{q}-g_{m}\right) / 3$. Let the $N$ bosons (with mass $m$ ) be confined by a harmonic trap with frequency $\omega$. When $\hbar \omega$ and $\sqrt{\frac{\hbar}{m \omega}}$ are used as units of energy and length, respectively, the Hamiltonian reads

$$
H=\frac{1}{2} \sum_{i}\left(-\nabla_{i}^{2}+r_{i}^{2}\right)+\sum_{i<j} U_{i j}
$$

Using the method as given in 15., the Hamiltonians for $N=3$ and 4 two-dimensional systems have been diagonalized. Both the orbital angular momentum $L$ and $S$ are good quantum numbers, therefore the eigenenergies and eigenstates can be denoted as $E_{L S}$ and $\Psi_{L S}$ (there is actually a series of states having the same $L$ and $S$, but only the lowest of them is concerned). It was found that, the levels are grouped as shown in Fig.1. In the lowest (first) group all bosons remain mostly in the lowest harmonic oscillator (h.o.) shell, in the second group one of the bosons is mainly in the first excited h.o. shell, etc. . All the levels in the first group have $L=0$, they would be degenerate if the interaction were spin-independent (i.e., $g_{q}=g_{m}$ or $c_{2}=0$ ), otherwise they would split into a band, the ground band (GB). All levels in the second group have $L=1$, they would be degenerate into two levels if $g_{q}=g_{m}$, and each would split into a band if $g_{q} \neq g_{m}$. Thus, the two lowest group contain totally three bands. In particular, we found that the third band is exactly a shift as a whole of the ground band by the unit $\hbar \omega$, therefore it is simply the c.m. excited states of the ground band. We shall concentrate on the second band, namely the FEB.

Since the total wave function must be totally symmetric with respect to particle permutation, it can be in general written as

$$
\Psi_{L S}=\sum_{k, \lambda j} f_{L S k, \lambda j} \vartheta_{S, k}^{\lambda j}
$$

where $\vartheta_{S, k}^{\lambda j}$ is the total spin-state with the good quantum numbers $S, S_{Z}$, and it is also the $j-t h$ basis function of the $\lambda$ representation of the $N$-body permutation group. The index $k$ refers to the multiplicity, i.e., more than one set of total spin states might have the same $S$ and $\lambda$. [16] $f_{L S k, \lambda j}$ is a spatial function belonging to the same representation $\lambda$. The summation over $j$ is necessary to assure $\Psi_{L S}$ to be totally symmetric. The summation over $\lambda$ and $k$ is necessary because that they are not conserved under the interaction. 
For a given state, from (2) we can define the weight of a representation as

$$
W_{L S, \lambda}=\sum_{k, j}<f_{L S k, \lambda j} \mid f_{L S k, \lambda j}>
$$

When $g_{q}=g_{m}$ the permutation symmetry is pure and therefore $W_{L S, \lambda}=1$ or 0 . When $g_{q} \neq g_{m}$, the mixing of $\lambda$ appears, but remains very weak. It was found that, for both $N=3$ and 4 , the GB is dominated by $\lambda=\{N\}$ , while the FEB by $\lambda=\{N-1,1\}$. Even if the spindependent interaction is very strong $\left(g_{q} / g_{m}=0.6\right.$ and $\left.g_{m}+g_{q}=3\right)$, the GB still has $W_{0 S,\{N\}} \geq 0.996$ and the FEB has $W_{1 S,\{N-1,1\}} \geq 0.998$, thus the mixing is still weak. Since the mixing is so slight, $\lambda$ is nearly a good quantum number. Therefore, the labels $(L, S, \lambda)$ can be used to label the states as marked in Fig.1.

There are four points noticeable.

(i) The zero-range interaction between two bosons would be zero if their spatial wave function is antisymmetric (because in this case the two bosons do not overlap). Since the interaction is repulsive, the representation $\lambda$ with more antisymmetric pairs is more advantageous to binding because the repulsion can be thereby reduced.

(ii) When one and only one boson has been excited to the second h.o. shell, the spatial permutation symmetry is restricted to $\lambda=\{N\}$ and $\{N-1,1\}$, and this explains why the second group contains two bands as shown in Fig.1. Where the band with $\{N-1,1\}$ is lower due to the reason stated in point (i). Thus the FEB would in general have $\lambda=\{N-1,1\}$.

(iii) The energy would also depend on $S$ due to the spin-dependent interaction. Thus, the levels with the same $\lambda$ would further split into a band.

(iv) When $g_{q}>g_{m}$, the pair coupled to $S_{i j}=2$ is more repulsive than the pair coupled to 0 . Evidently, the many-body state with a larger $S$ would have more pairs coupled to 2 . Therefore, in a band, the state with a larger $S$ is higher. On the contrary, when $g_{q}<g_{m}$, the state with a larger $S$ is lower as shown in Fig.1.

To generalize the above study to the case with a very large $N$, we need the knowledge of the total spinstates. For the most important case of $\lambda=\{N\}$, there is an unique $\vartheta_{S, k}^{\{N\} j}$ if $N-S$ is even, or there is none otherwise 14, 17]. Therefore both the labels $j$ and $k$ are not necessary, and a simpler notation $\vartheta_{S}^{\{N\}} \equiv \vartheta_{S, k}^{\{N\} j}$ can be used.

Let us start from a 2-boson system with $\vartheta_{S}^{\{2\}}(12)=$ $(\chi(1) \chi(2))_{S}$, where $\chi(i)$ is the single boson spin-state, $S=0$ or 2 . For $N=3$, the requirement that $N-S$ is even leads to $S=3$ and 1 . They were given in [15] as

$$
\begin{gathered}
\vartheta_{3}^{\{3\}}=\left\{(\chi(1) \chi(2))_{2} \chi(3)\right\}_{3}=\left\{\vartheta_{2}^{\{2\}} \chi(3)\right\}_{3} \\
\vartheta_{1}^{\{3\}}=\frac{2}{3}\left\{\vartheta_{2}^{\{2\}} \chi(3)\right\}_{1}+\frac{\sqrt{5}}{3}\left\{\vartheta_{0}^{\{2\}} \chi(3)\right\}_{1}
\end{gathered}
$$

Starting from (44) and (5), let us assume that all $\vartheta_{S^{\prime}}^{\{N-1\}}$ of the $(N-1)$-boson system have been known. Then, taking the rule of angular momentum coupling and the rule of outer product into account, the $\vartheta_{S}^{\{N\}}$ of the $N$-boson system can be uniquely expanded as

$$
\vartheta_{S}^{\{N\}}=a_{S}^{\{N\}}\left\{\vartheta_{S+1}^{\{N-1\}} \chi(N)\right\}_{S}+b_{S}^{\{N\}}\left\{\vartheta_{S-1}^{\{N-1\}} \chi(N)\right\}_{S}
$$

where $a_{S^{\prime}}^{\{N\}}$ and $b_{S^{\prime}}^{\{N\}}$ are the fractional parentage coefficients (FPC) to be determined, however they are zero if $N-S$ is odd.

From (6), a similar formula can be written for $\vartheta_{S \pm 1}^{\{N-1\}}$ - Therefore, $\vartheta_{S}^{\{N\}}$ can be expanded once more as

$$
\begin{aligned}
\vartheta_{S}^{\{N\}} & =a_{S}^{\{N\}} a_{S+1}^{\{N-1\}}\left\{\left[\vartheta_{S+2}^{\{N-2\}} \chi(N-1)\right]_{S+1} \chi(N)\right\}_{S} \\
& +a_{S}^{\{N\}} b_{S+1}^{\{N-1\}}\left\{\left[\vartheta_{S}^{\{N-2\}} \chi(N-1)\right]_{S+1} \chi(N)\right\}_{S} \\
& +b_{S}^{\{N\}} a_{S-1}^{\{N-1\}}\left\{\left[\vartheta_{S}^{\{N-2\}} \chi(N-1)\right]_{S-1} \chi(N)\right\}_{S} \\
& +b_{S}^{\{N\}} b_{S-1}^{\{N-1\}}\left\{\left[\vartheta_{S-2}^{\{N-2\}} \chi(N-1)\right]_{S-1} \chi(N)\right\}_{S}
\end{aligned}
$$

On the other hand, since the $\vartheta_{S}^{\{N\}}$ is totally symmetric as required, the right hand side of (7) must be invariant against the interchange of the $\mathrm{N}$-th and (N-1)-th bosons. This leads to a set of three homogeneous equations. It turns out that they are linearly dependent, one of them reads

$$
\begin{aligned}
& {\left[\hat{W}_{S}(S+1, S+1)-1\right] a_{S}^{\{N\}} b_{S+1}^{\{N-1\}} } \\
+ & \hat{W}_{S}(S-1, S+1) b_{S}^{\{N\}} a_{S-1}^{\{N-1\}}=0
\end{aligned}
$$

where

$$
\hat{W}_{S}\left(T, T^{\prime}\right)=\sqrt{(2 T+1)\left(2 T^{\prime}+1\right)} W\left(1 S S 1 ; T T^{\prime}\right)
$$

and $W\left(1 S S 1 ; T T^{\prime}\right)$ is the well known Racah coefficients. From (8) we obtain

$$
\begin{gathered}
a_{S}^{\{N\}}=\gamma_{S}^{\{N\}} \frac{\sqrt{2 S-1}}{S \sqrt{2 S+1}} / b_{S+1}^{\{N-1\}} \\
b_{S}^{\{N\}}=\gamma_{S}^{\{N\}} \frac{\sqrt{2 S+3}}{(S+1) \sqrt{2 S+1}} / a_{S-1}^{\{N-1\}}
\end{gathered}
$$

if $N-S$ is even, where the constant $\gamma_{S}^{\{N\}}$ is introduced to assure $\left(a_{S}^{\{N\}}\right)^{2}+\left(b_{S}^{\{N\}}\right)^{2}=1$, so that $\vartheta_{S}^{\{N\}}$ is normalized. Eqs.(10) and (11) together with the condition of normalization lead to a unique solution, thus we obtain the analytical form of FPC as

$a_{S}^{\{N\}}=\left[\left(1+(-1)^{N-S}\right)(N-S)(S+1) /(2 N(2 S+1))\right]^{1 / 2}$

$$
b_{S}^{\{N\}}=\left[\left(1+(-1)^{N-S}\right) S(N+S+1) /(2 N(2 S+1))\right]^{1 / 2}
$$


and

$$
\gamma_{S}^{\{N\}}=S(S+1)\left[\frac{(N-S)(N+S+1)}{N(N-1)(2 S+3)(2 S-1)}\right]^{1 / 2}
$$

The FPC are very useful because related matrix elements among the total spin-states can be thereby calculated.

Now let us study the set of total spin-states with the $\{N-1,1\}$ symmetry. It has been proved that the multiplicity of this set is one [16], therefore they can be labelled in a simpler way as $\Theta_{S}^{i} \equiv \vartheta_{S, k}^{\{N-1,1\} i}$. Since the dimension of $\{N-1,1\}$ representation is $N-1, i=1,2, \cdots, N-1$ . Making use of the FPC, they can be expanded as

$$
\Theta_{S}^{i}=b_{S}^{\{N\}}\left\{\vartheta_{S+1}^{\{N-1\}}(\stackrel{\times}{i}) \chi(i)\right\}_{S}-a_{S}^{\{N\}}\left\{\vartheta_{S-1}^{\{N-1\}}(\stackrel{\times}{i}) \chi(i)\right\}_{S}
$$

if $N-S$ is even, and

$$
\Theta_{S}^{i}=\left\{\vartheta_{S}^{\{N-1\}}(\stackrel{\times}{i}) \chi(i)\right\}_{S}
$$

if $N-S$ is odd, where the notation $\stackrel{\times}{i}$ represents all the bosons except the $i-t h$. From the rule of outer product, (15) and (16) will contain only the symmetries $\{N\}$ and $\{N-1,1\}$. However, when $N-S$ is even, (15) is orthogonal to the unique totally symmetric spinstate $\vartheta_{S}^{\{N\}}$. Hence, it has the pure $\{N-1,1\}$ symmetry. When $N-S$ is odd, it has been stated above that $\vartheta_{S}^{\{N\}}=$ 0 , therefore (16) has also the pure $\{N-1,1\}$ symmetry. It is obvious from (12) and (13) that $\Theta_{S}^{i}$ does not exist if $S=0$ or $N$. Thus the $S$ of $\Theta_{S}^{i}$ is ranged from 1 to $N-1$ , while the index $i$ of $\Theta_{S}^{i}$ is ranged from 1 to $N$. This set of $N$ states are not mutually orthogonal but linearly dependent. They satisfy $\sum_{i} \Theta_{S}^{i}=0$. This arises because the summation over $i$ leads to a symmetrization, a state with $\lambda \neq\{N\}$ will not survive after the symmetrization.

Based on the knowledge of $\Theta_{S}^{i}$, we can begin to study the FEB (three-dimensional) of realistic cases. Let the normalized single-particle state of condensation be denoted as $\varphi_{S}^{a}=\frac{1}{\sqrt{4 \pi} r} u_{S}^{a}(r)$, while the excited state be denoted as $\varphi_{S}^{b}=\frac{1}{r} u_{S}^{b}(r) Y_{l m}(\theta, \phi)$ with $l=1$. As a basic assumption we define the spatial wave function as

$$
F_{S, i}=\varphi_{S}^{b}(i) \Pi_{j(\neq i)} \varphi_{S}^{a}(j)
$$

Then, the total wave function is written as

$$
\Psi_{S}=\sum_{i} F_{S, i} \Theta_{S}^{i}
$$

Where the summation over $i$ leads to a symmetrization as required, and the $\{N-1,1\}$-representation has been introduced. The expansion in (18) is different from (2), but both are eigenstates of the total spin and symmetrical with respect to particle permutation, thus both are applicable.

Inserting (18) into the Schrödinger equation, using the standard variational procedure, the equations for $\varphi_{S}^{a}$ and
$\varphi_{S}^{b}$ can be deduced as

$$
\begin{gathered}
{\left[h_{0}+g_{S}^{b} \frac{\left|u_{S}^{b}\right|^{2}}{4 \pi r^{2}}+(N-2) g_{S}^{a} \frac{\left|u_{S}^{a}\right|^{2}}{4 \pi r^{2}}\right] u_{S}^{a}=\varepsilon_{a} u_{S}^{a}} \\
{\left[h_{1}+(N-1) g_{S}^{b} \frac{\left|u_{S}^{a}\right|^{2}}{4 \pi r^{2}}\right] u_{S}^{b}=\varepsilon_{b} u_{S}^{b}}
\end{gathered}
$$

where

$$
h_{0}=\frac{1}{2}\left[-\frac{d^{2}}{d r^{2}}+r^{2}\right]
$$

$$
h_{1}=\frac{1}{2}\left[-\frac{d^{2}}{d r^{2}}+\frac{2}{r^{2}}+r^{2}\right]
$$

$$
g_{S}^{b}=\left\langle\Theta_{S}^{i}\left|O_{i j}\right| \Theta_{S}^{i}+\Theta_{S}^{j}\right\rangle
$$

and

$$
g_{S}^{a}=\left\langle\Theta_{S}^{i}\left|O_{j k}\right| \Theta_{S}^{i}\right\rangle
$$

The energies of the states in the FEB are given by

$$
\begin{aligned}
E_{S}^{(e)} & =\varepsilon_{b}+(N-1) \varepsilon_{a} \\
& -\frac{(N-1) g_{S}^{b}}{4 \pi} \int_{0}^{\infty} \frac{d r}{r^{2}}\left|u_{S}^{a}\right|^{2}\left|u_{S}^{b}\right|^{2} \\
& -\frac{(N-1)(N-2) g_{S}^{a}}{8 \pi} \int_{0}^{\infty} \frac{d r}{r^{2}}\left|u_{S}^{a}\right|^{4}
\end{aligned}
$$

where $S$ can be even or odd, and is ranged from 1 to $N-1$.

Using the expansions (15) and (16) twice (one is for the $N-1$ body system), with the FPC given by (12) and (13), the matrix elements among $\Theta_{S}^{i}$ can be obtained as

$$
\begin{aligned}
g_{S}^{b}=\frac{N-2}{N-1}\left(c_{0}+c_{2}\right)-\left(1+(-1)^{N-S}\right) \frac{S(S+1)}{N(N-1)} c_{2} \\
g_{S}^{a}=c_{0}+c_{2} \frac{1}{(N-1)(N-2)} . \\
{\left[\frac{N+1+(-1)^{N-S}}{N} S(S+1)-2(N-1)\right] }
\end{aligned}
$$

In the FEB, all the members with odd $N-S$ do not have their partners in the ground band, they are the lowest states with the given $S$. If the system is cooled from higher to zero temperature, and if no spin-relaxation mechanism is available, the probability of staying in the FEB is nearly half. Incidentally, when $N$ is large, since $g_{S}^{a}$ is very close to the $g_{S}=$ $c_{0}+(S(S+1)-2 N) /(N(N-1)) c_{2}$ of the ground band ${ }^{14}$ , thus $\varphi_{S}^{a}$ is very close to that of the ground band if $N-S$ is even. 
As an application we calculated the FEB of ${ }^{23} \mathrm{Na}$ atoms trapped in a harmonic well with $\hbar \omega=100 \mathrm{~Hz}$ and with $g_{m}=6.351 \times 10^{-4} \sqrt{\omega}, g_{q} / g_{m}=1.10$. The normalized radial wave functions $R^{a}(r)=u_{S}^{a}(r) / r$ and $R^{b}(r)=u_{S}^{b}(r) / r$ are given in Fig.2 (a) and (b) respectively.

Let $E_{S}^{(g)}$ denote the energies of the $\mathrm{GB}\left(E_{0}^{(g)}\right.$ is the ground state energy ). The upper and lower bounds of the FEB, i.e., $E_{N-1}^{(e)}$ and $E_{1}^{(e)}$ relative to $E_{0}^{(g)}$ are plotted in Fig.3. This figure demonstrates how the FEB and GB split. In particular when $N$ gets larger, the lower bound of FEB would cross the upper of the GB, it implies an overlap of these two bands.

In summary, we have derived a set of equations to describe the first excited band, this might lead to a deeper understanding of the condensates at very low temperature. Furthermore, we have achieved the analytical form of the fractional parentage coefficients. This will greatly facilitate the calculations dealing with the total spin-states, and will thereby promote the applicability of the S-conserved theory for various spinor many-body systems.

Acknowledgment; We appreciate the support by NSFC under the grants 90103028 and 90306016 .

[1] A. Einstein, Sitzungsber. Preuss. Akad. Wiss., Phys. Math. Kl. 1925, 3 (1925)

[2] M.H. Anderson, J.R. Ensher, M.R. Matthews, C.E. Wieman, and E.A. Cornell, Science 269, 198 (1995)

[3] K.B. Davis, M.-O. Mewes, and W. Ketterle, Appl. Phys. B: Laser Opt. B60, 155 (1995)

[4] C.C. Bradley, C.A. Sackett, J.J. Tollett, and R.G. Hulet, Phys. Rev. Lett. 75, 1687 (1995); 79, 1170 (1997)

[5] D.M. Stamper-Kurn, M.R. Andrews, A.P. Chikkatur, S. Inouye, H.-J. Miesner, J. Stenger, and W. Ketterle, Phys. Rev. Lett. 80, 2027 (1998)

[6] L.-M. Duan, J.I. Cirac, and P. Zoller, Phys. Rev. A 65, 033619 (2002)

[7] C.K. Law, H. Pu, and N.P. Bigelow, Phys. Rev. Lett. 81, 5257 (1998)

[8] E. Goldstein and P. Meystre, Phys. Rev. A 59, 3896 (1998)

[9] T.-L. Ho and S.K. Yip, Phys. Rev. Lett. 84, 4031 (2000)

[10] M. Koashi, and M. Ueda, Phys. Rev. Lett. 84, 1066 (2000)

[11] S. Yi, Ö. E. Müstecaphoğlu, C.P. Sun, and L. You, Phys. Rev. A 66, 011601R (2002)

[12] S. Ashhab and A.J. Leggett, Phys. Rev. A 68, 063612 (2003)

[13] A.A. Svidzinsky and S.T. Chui, Phys. Rev. A 68, 043612 (2003)

[14] C.G. Bao and Z.B. Li, Phys. Rev. A. 70, 043620 (2004)

[15] C.G. Bao and T.Y. Shi, Phys. Rev. A, 68, 032509 (2003); 66, 013613 (2002)

[16] J. Katriel, J. of Molecular Structure (Theo. Chem.), 547, 1 (2001)

[17] Y. Wu, Phys. Rev. A 54, 4534 (1996). 


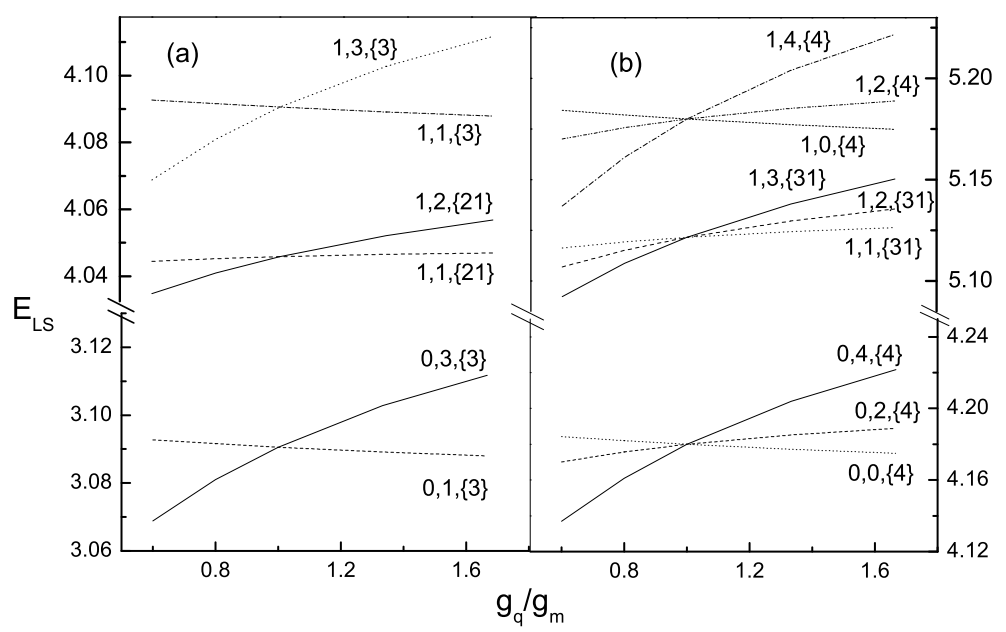

FIG. 1: The evolution of $E_{L S}$ (in $\hbar \omega$ ) with $g_{q} / g_{m}$ in 3 -boson (a) and 4-boson (b) systems. $(L, S, \lambda)$ are marked by the levels. 


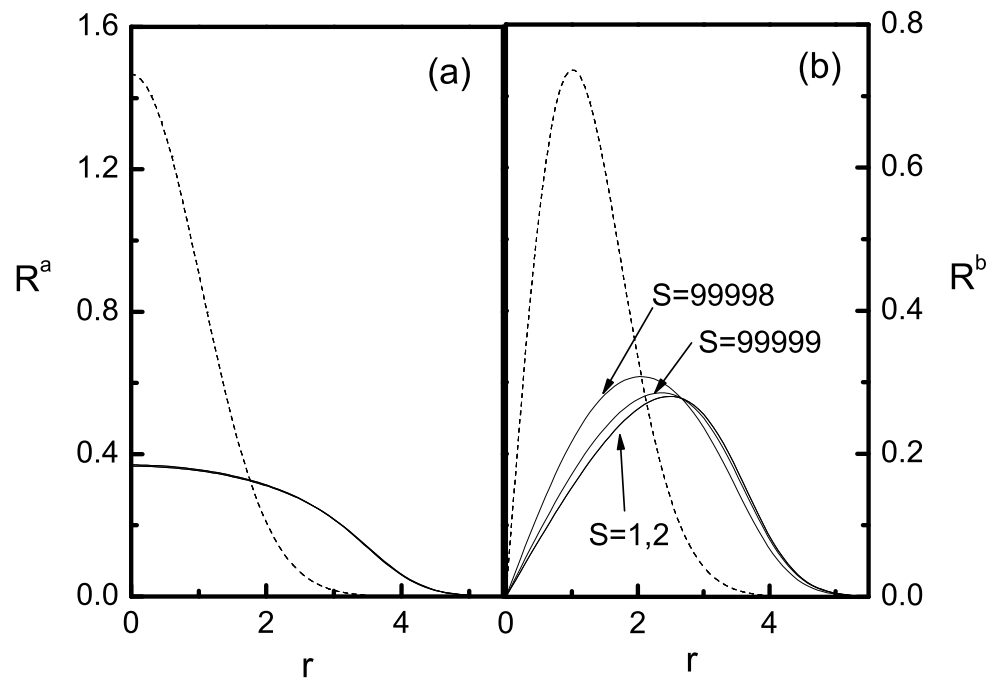

FIG. 2: The radial wave functions, $R^{a}$ (in (a)) and $R^{b}$ (in (b) ), as functions of $r$. The dashed curves are for $N=100$, while the solid ones are for $N=10^{5}$. In each case the curves for $S=N-1, N-2,2$, and 1 are plotted. However, they can not be distinguished except the case of $R^{b}$ with a large $N$, where $S$ are marked by the curves. In (b), when $S$ lies in between and $N-S$ is even (odd), the associated curves are distributed between the curves with $S=2$ and 99998 (1 and 99999 ). Obviously, when $N-S$ is odd, the S-dependence is less explicit, and the $R^{b}$ with $S=N-2$ has a smallest size.

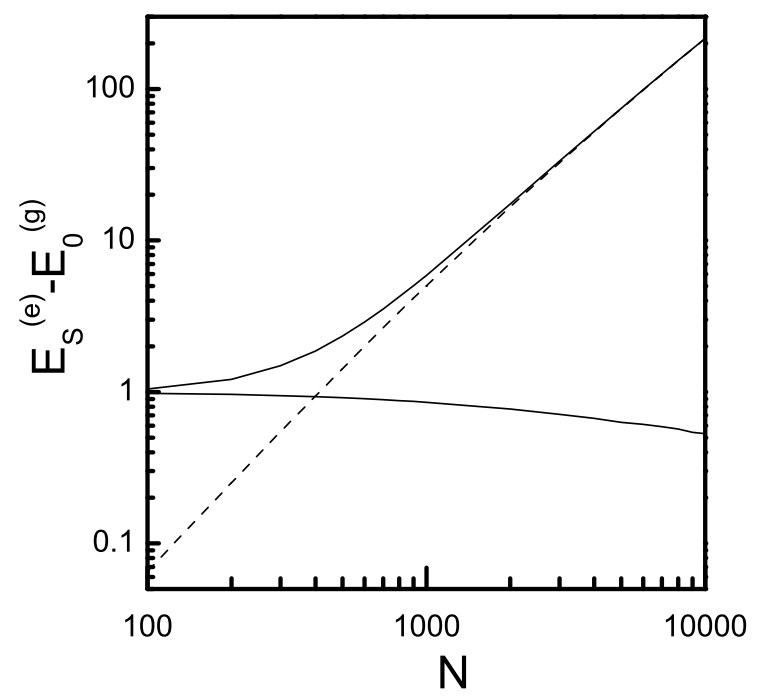

FIG. 3: $E_{S}^{(e)}-E_{0}^{(g)}$ against $N$. The upper (lower) solid line has $S=N-1(1)$. The dashed line is for $E_{N}^{(g)}-E_{0}^{(g)}$, namely the split of the GB. 